\title{
Titanium-Based Microbolometers: Control of Spatial Profile of Terahertz Emission in Weak Power Sources
}

\author{
Linas Minkevičius ${ }^{1, *(\mathbb{D}}$, Liang $Q i^{1}{ }^{\mathbb{D}}$, Agnieszka Siemion $\left.{ }^{2} \mathbb{(}\right)$, Domas Jokubauskis ${ }^{1}(\mathbb{D}$, \\ Aleksander Sešek ${ }^{3}{ }^{\circledR}$, Andrej Švigelj ${ }^{3}$, Janez Trontelj ${ }^{3}$, Dalius Seliuta ${ }^{1}$, Irmantas Kašalynas ${ }^{1}$ (D) \\ and Gintaras Valušis ${ }^{1}$ (i) \\ 1 Department of Optoelectronics, Center for Physical Sciences and Technology, Saulètekio Ave. 3, \\ 10257 Vilnius, Lithuania; liang.qi@ftmc.lt (L.Q.); domas.jokubauskis@ftmc.lt (D.J.); \\ dalius.seliuta@ftmc.lt (D.S.); irmantas.kasalynas@ftmc.lt (I.K.); gintaras.valusis@ftmc.lt (G.V.) \\ 2 Faculty of Physics, Warsaw University of Technology, 75 Koszykowa, 00662 Warsaw, Poland; \\ agnieszka.siemion@pw.edu.pl \\ 3 Laboratory for Microelectronics, Faculty of Electrical Engineering, University of Ljubljana, Tržaška 25, \\ 1000 Ljubljana, Slovenia; aleksander.sesek@fe.uni-lj.si (A.S.); andrej.svigelj@fe.uni-lj.si (A.Š.); \\ janez.trontelj1@guest.arnes.si (J.T.) \\ * Correspondence: linas.minkevicius@ftmc.lt; Tel.: +370-5-243-1200
}

Received: 17 April 2020; Accepted: 11 May 2020; Published: 14 May 2020 updates

Featured Application: Convenient and easy-to-use detectors for accurate adjustment and control of spatial mode profiles without additional focusing components for various weak power $\mathrm{THz}$ sources operating either in time-domain or continuous wave regimes.

Abstract: Terahertz (THz) imaging and spectroscopy set-ups require fine optical alignment or precise control of spatial mode profile. We demonstrate universal, convenient and easy-to-use imaging — resonant and broadband antenna coupled ultrasensitive titanium-based-dedicated to accurately adjust and control spatial mode profiles without additional focusing optical components of weak power $\mathrm{THz}$ sources. Versatile operation of the devices is shown using different kinds of $\mathrm{THz}$-electronic multiplier sources, optical $\mathrm{THz}$ mixer-based frequency domain and femtosecond optoelectronic THz time-domain spectrometers as well as optically pumped molecular THz laser. Features of the microbolometers within $0.15-0.6 \mathrm{THz}$ range are exposed and discussed, their ability to detect spatial mode profiles beyond the antennas resonances, up to $2.52 \mathrm{THz}$, are explored. Polarization-sensitive mode control possibilities are examined in details. The suitability of the resonant antenna-coupled microbolometers to resolve low-absorbing objects at $0.3 \mathrm{THz}$ is revealed via direct, dark field and phase contrast imaging techniques as well.

Keywords: microbolometer; terahertz sensing; terahertz imaging systems; dark field method; phase contrast method

\section{Introduction}

Terahertz (THz) imaging and spectroscopy revealed itself as promising technologies for a large variety of applications [1]. Because of non-ionizing nature of $\mathrm{THz}$ radiation, imaging in this range can be found as a beneficial tool for medicine and biomedical stuff inspection [2-4]. As THz frequencies display the ability to propagate in dielectric medium without strong attenuation, $\mathrm{THz}$ imaging and spectroscopy can also serve as valuable instruments for security needs [5-7], post packages inspection [8-10] or industrial materials control and their identification [11-14]. However, direct implementation of $\mathrm{THz}$ technology still meets severe difficulties related mainly to reliability of active components (emitters and detectors) and issues of precise alignment of the optical system due to the 
presence, as a rule, of passive bulky optical elements like mirrors, lenses or beam splitters. Thus, effective and convenient operation under real environmental conditions still needs to overcome alignment and compact optics problems [15]. On the other hand, it is of decisive demand to provide adequate and favourable control of the spatial mode profile of illumination. In particular, it has strong impact in $\mathrm{THz}$ spectroscopy for quality of recorded $\mathrm{THz}$ spectra in increasing its bandwidth, lowering the noise floor, as well as by reducing uncertainties in observational data [16-18]. It displays also important role in $\mathrm{THz}$ imaging, especially, in examining bulky objects when extraordinary means are needed to be applied in $\mathrm{THz}$ beam engineering aiming to provide appropriate image quality $[19,20]$.

Spatial mode profiles of discrete $\mathrm{THz}$ spectrum radiation from backward wave oscillators and quantum cascade lasers were already studied by employing thin film vanadium oxide (VOx) based bolometers [21]. Moreover, image of the beam emitted by an optoelectronical $\mathrm{THz}$ time-domain spectrometer was recorded using a camera integrating an array of $\mathrm{THz}$ optimized antenna-coupled amorphous silicon-based microbolometers [22]. Indeed, it is of distinct interest as $\mathrm{THz}$ time-domain spectrometers become widespread, they require precise alignment to get well collimated THz beams, hence, it is beneficial to engage easy-to-use imaging detector to accurately adjust these optical systems. The sensitivity of the presented microbolometers allowed to register images of spatial mode profiles as well as video images despite the fact that their average power was in the range of a few $\mu \mathrm{W}$ [22].

The developed and described here antenna-coupled titanium-based microbolometer (Ti-mB) [23] was initially applied in compact $\mathrm{THz}$ imaging systems for security aims [24,25]. Its high sensitivity was successfully exploited also in medical applications via direct THz imaging of carcinoma affected tissues [26].

In a given work, by coupling of the titanium microbolometers with relevant resonant antennas, we modified these sensitive detectors into universal, convenient and easy-to-use imaging instruments well-suited to accurately adjust weak power $\mathrm{THz}$ sources without additional focusing components. They can be employed in imaging systems operating either in time-domain or in continuous wave modes for precise alignment and control of their spatial mode profiles. Versatile operation of the devices is displayed engaging different kinds of THz emitters - electronic multiplier sources (THz ELS), optical $\mathrm{THz}$ mixer-based frequency domain (THz FDS) and femtosecond optoelectronic $\mathrm{THz}$ time domain (THz TDS) spectrometers as well as optically pumped molecular THz laser (THz OPML). Features of the microbolometers coupled with resonant antennas in subTHz range, within the $0.15-0.6 \mathrm{THz}$ range, are exposed and discussed here. The ability to monitor spatial mode profile even far above the antennas resonances, up to $2.52 \mathrm{THz}$, is explored. Moreover, the possibilities of polarization-sensitive mode control are examined in details, and the recorded mode control results are compared with that obtained using commercially available pyroelectric sensors. In addition, the suitability of the resonant antenna coupled Ti-mB to resolve low-absorbing objects at $0.3 \mathrm{THz}$ is revealed via direct, dark field and phase contrast imaging techniques.

\section{Bolometers' Design and Experimental Set-Ups}

The design of antenna-coupled Ti-mB and its cross section are presented in Figure 1. As it can be seen, the device is constructed using air-bridged approach. The dual dipole $\mathrm{THz}$ antenna is coupled to the bolometer and serves as a filter that aims to efficiently collect the incident $\mathrm{THz}$ radiation and convert its energy to temperature change. The Ti-mB and $\mathrm{THz}$ antenna were processed on a few microns thin SiN membrane to enhance the sensitivity and the response time via reduction of thermal losses of the instrument. The metalized bottom plate under the antenna-bolometer acts as reflecting mirror, additionally amplifying the received signal on the dipole. More detailed description on the design and the technology used to manufacture such devices can be found in Refs. [23,24]. 


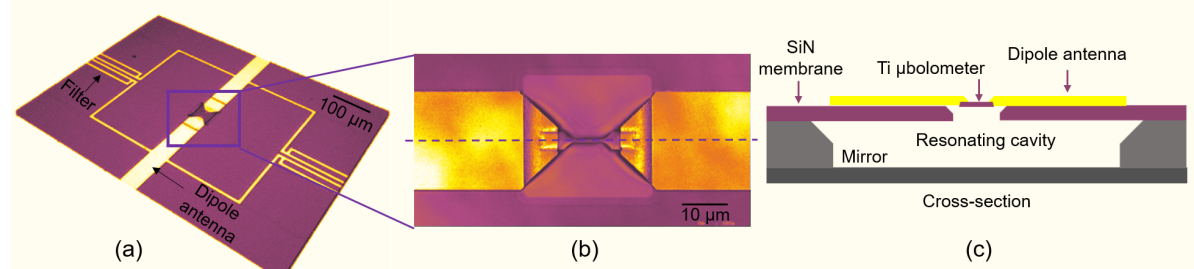

Figure 1. The photo of the designed terahertz titanium-based microbolometer with antenna and filters (a). The enlarged view of the bridge active part of the microbolometer: the antenna area is approximately $500 \mu \mathrm{m} \times 500 \mu \mathrm{m}$, the geometry of visible titanium bridge amounts to $12 \mu \mathrm{m}$ length and $2 \mu \mathrm{m}$ width (b). The cross-section of microbolometric detector. Note that the selectivity was enhanced by adjustment of the dipole antenna geometry and the resonant-cavity design: back side reflection mirror was positioned at the quarter wavelength distance (c). Adapted from Refs. [23,24].

Experimental set-ups for terahertz beam profiles imaging and their control are shown in Figure 2. All experiments were performed using raster scanning technique with one pixel detector from linear array of 4 or 32 pixels to obtain beam profile images in $x y$ plane. The measuring time per pixel in most experiments was in between $10 \mathrm{~ms}$ and $20 \mathrm{~ms}$. The technical details on used equipment and measurement techniques are presented in Section 4.
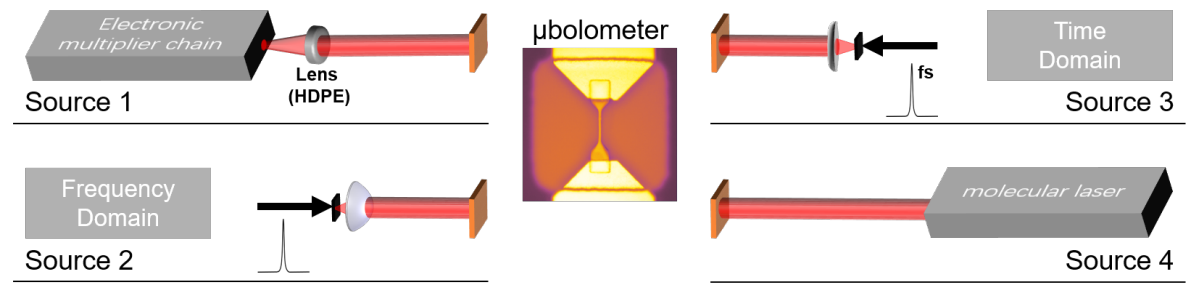

Figure 2. Experimental set-up for imaging of terahertz beam profiles using different $\mathrm{THz}$ emitters. Source 1-electronic multiplier chain emitting radiation of $0.15 \mathrm{THz}, 0.3 \mathrm{THz}$ and $0.6 \mathrm{THz}$ frequencies in continuous wave mode. Source 2-low temperature grown galium arsenide (LTG GaAs) photo-mixer coupled with silicon lens in frequency domain spectrometer delivering radiation from $0.1-2 \mathrm{THz}$. Source 3-photoconductive antenna on LTG GaAs from time domain spectrometer generating broadband radiation up to $2.5 \mathrm{THz}$. Source 4-optically pumped continuous wave molecular THz laser emitting the discrete spectrum at $0.76 \mathrm{THz}, 1.63 \mathrm{THz}$ and $2.52 \mathrm{THz}$. Center panel displays photo of Ti-based microbolometer.

\section{Measurement Results and Discussion}

Conventional the diagnostic tool to investigate the THz beam's spatial profile is typical beam imaging, where radiation intensity is scanned in the plane perpendicular to the direction of propagation. To measure the beam profile, focusing optics is usually used to concentrate $\mathrm{THz}$ radiation aiming to increase the detected signal. However, any additional focusing element in beam path can distort the beam profile and in many cases cause unwanted interferences. In the $\mathrm{THz}$ range, it is of particular importance to use off-axis parabolic mirrors, but then small misalignment can strongly affect the beam profile. As an alternative, one can use plastic lenses; however, they display feature to change focus distance depending on the frequency of incident radiation and they are not so attractive in broadband THz TDS systems, where frequency range, as a rule, extends by two orders of magnitude. To measure the beam profile correctly, one needs to scan unfocused beam that requires high responsivity $\mathrm{THz}$ sensor to have sufficient signal-to-noise ratio (SNR). The use of a coherent detector in such a case is limited because the $\mathrm{Si}$ lens coupled detector yields limited imaging resolution. Usually, one can employ conventional variable size aperture scanning and Hartmann test techniques to measure far field beam profile [16]. One can note that such approaches are easy to apply and do not require reconfiguration of set-up, nonetheless, reaching high spatial resolution, especially for high $\mathrm{THz}$ frequencies, can be rather 
complicated because it is determined by the aperture size, and its reduction, consequently, induces the decrease in SNR.

\subsection{Mode Control in Electronic Multiplier Sources}

Initially, the focus of the investigation was attributed to spatial features of $\mathrm{THz}$ radiation delivered from electronic multiplier sources. One can note that profile was artificially perturbed by detuning the source from the optimal working regime. The results are shown in Figure 3, where the performance of Ti-mB to record a continuous wave $(\mathrm{CW})$ mode is demonstrated for different polarizations and at three selected frequencies $0.6 \mathrm{THz}, 0.3 \mathrm{THz}$ and $0.15 \mathrm{THz}$. For comparison, measurement results registered by the pyroelectric detector are illustrated in a panel (a) of Figure 3, while the beam profiles at different polarizations are presented in panels (b) and (c). Pyroelectric detector in principle does not detect the polarization of the incident light, thus, registered signal represents total power value of the $\mathrm{THz}$ radiation. Despite this fact, the signal values induced in microbolometer and presented in panel (b) are 4-7 times larger than those in panel (a) if compared within the same frequency. In contrast, the microbolometer is polarization-sensitive, thus, not only the mode profiles, but also their fine structure are clearly resolved, which is visible in Figure $3 \mathrm{~b}, \mathrm{c}$. Comparing the polarization extinction ratio (PER), it amounts to $11 \mathrm{~dB}, 11.8 \mathrm{~dB}$ and $7.8 \mathrm{~dB}$ at $0.6 \mathrm{THz}, 0.3 \mathrm{THz}, 0.15 \mathrm{THz}$, respectively, which indicates the suitability of the Ti-mB for polarization-sensitive applications. Special emphasis is required to the results obtained at $0.15 \mathrm{THz}$ (panel (c)). Estimates show that the microbolometer connecting wires can act as additional coupling antennas disturbing consequently precise control of the radiation polarization and the mode shape.

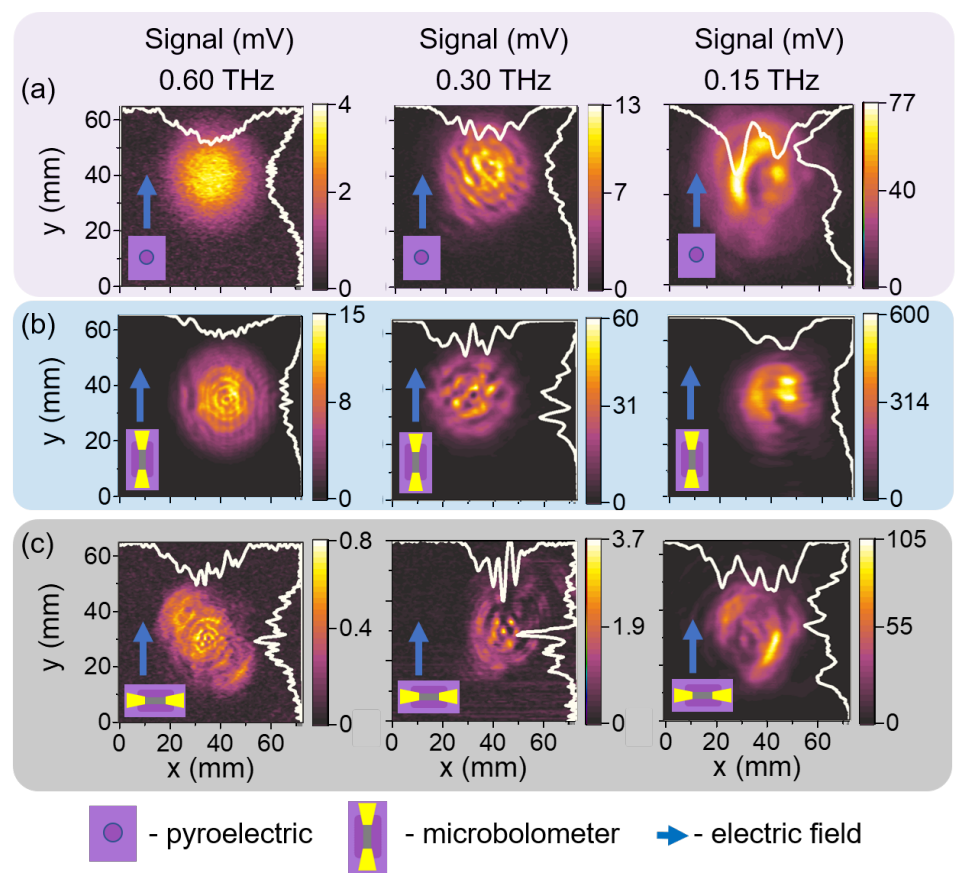

Figure 3. Scheme of polarisation orientation and $\mathrm{THz}$ beam profiles obtained using electronic multiplier chain source operating in a continuous wave $(\mathrm{CW})$ mode at different frequencies: $0.6 \mathrm{THz}, 0.3 \mathrm{THz}$ and $0.15 \mathrm{THz}$. Reference measurements using pyroelectric detector (a); results using microbolometer when the dipole antenna is in parallel to the electric field of the incident light (b); results using microbolometer when the dipole antenna is perpendicular to the electric field of the incident light (c). White lines indicate intensity cross sections along the relevant axes.

\subsection{Mode Control in Frequency Domain Spectrometer}

One of the important issues to record qualitative spectra, is the precise alignment of the spectroscopic or imaging systems. To illustrate suitability of Ti-mB for such aims, we have performed 
a special study using optical mixer-based frequency domain spectrometer. The THz FDS is operating within the frequency range $0.1-2 \mathrm{THz}$ and it delivers radiation of circular polarization of about $1 \mu \mathrm{W}$ power at $0.2 \mathrm{THz}$. Therefore, no exceptional attention was attributed to the polarization-related features; however, we restricted ourselves to subTHz range, close to the designed antennas resonances. The mode profile was disturbed intentionally to reveal abilities of the device in recording spatial mode structure. The mode profile was not corrected intentionally. A linear array of the bolometers coupled with antennas exhibiting resonances around $0.2 \mathrm{THz}$ and $0.4 \mathrm{THz}$ was investigated. The results of the measurements are presented in Figure 4. As it is seen, spatial mode profiles are clearly resolved, in particular, at $0.2 \mathrm{THz}$ (panel (a)), where delivered power is close to the maximal value.
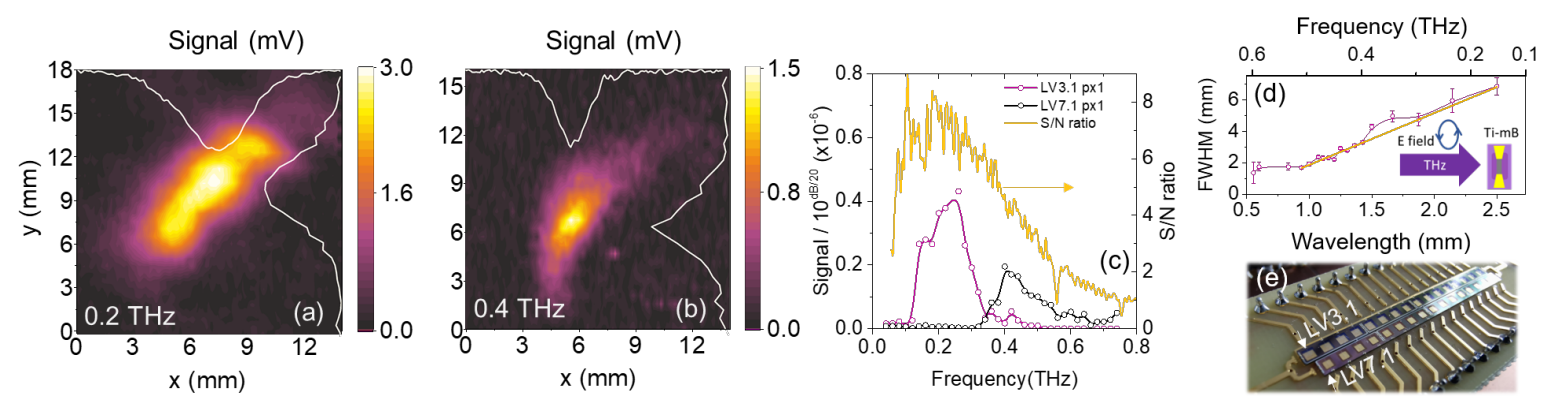

Figure 4. THz beam spatial profiles emitted by low-temperature grown GaAs-based emitter in frequency domain spectrometer at $0.2 \mathrm{THz}(\mathbf{a})$ and $0.4 \mathrm{THz}(\mathbf{b})$ recorded using a linear array of the bolometers coupled with antennas. White lines indicate intensity cross sections along the relevant axes. Spectral characteristics of the detectors with different resonant dipole antennas and spectra normalized to the spectrometer signal-to-noise ratio (SNR) (c); full-width half-maximum (FWHM) dependency on the wavelength with linear approximation depicted by the dark yellow line (d), configuration of measurement scheme and polarization are depicted in the inset of (d). A photo of dual frequency $\mathrm{THz}$ linear array, where the top line of pixels is designed for frequencies around $0.2 \mathrm{THz}$ and the bottom line for frequencies around $0.4 \mathrm{THz}$. The pixel pitch is $2 \mathrm{~mm}(\mathbf{e})$.

Moreover, the frequency selected exactly falls into relatively broad antenna resonance. The spatial profile presented at $0.4 \mathrm{THz}$ (Figure $4 \mathrm{~b}$ ) is less expressed. It was intentionally done aiming to highlight the convenience and universal operation of the device by detuning the emission frequency to the red side of the spectrum of the antenna resonance as can be evident from panel (c). Despite this fact, the spatial profile can still be precisely controlled although the power absorbed by the microbolometer is significantly reduced. This advantage can further be exploited in determining and monitoring spectral properties of frequency domain spectrometer. It can be illuminated, for instance, via determination of full-width half-maximum (FWHM) dependency on the wavelength of the emission. The results are depicted in Figure 4d. As one can see, the FWHM increases with the wavelength, and the dependence can be approximated by a linear law represented by a dark yellow line (Figure 4d). Thus, Ti-mB microbolometers and their linear array (Figure 4e) can manifest themselves as powerful instruments in fine alignment and control in spectrometers employing weak power $\mathrm{CW} \mathrm{THz}$ sources.

\subsection{Mode Control in Time-Domain Spectrometer}

In the context of the obtained results in $\mathrm{THz}$ frequency-domain, it is reasonable to turn the research into time-domain spectrometry and check a suitability of Ti-mB for this kind of THz systems. In contrast to frequency domain, inherent feature of time-domain spectroscopic systems is their broadband radiation; on the other hand, power of the radiation is in the same range as in THz FDS, i.e., of a few microwatts. Furthermore, THz TDS systems require precise alignment to measure spectra correctly or record images of acceptable quality. In both cases the polarisation of the incident light was parallel to the bolometer axis. 
To reveal picture of operation, two types of microbolometers were designed for the experiments: the first one was coupled with resonant antennas for $0.3 \mathrm{THz}$ and the second for $0.7 \mathrm{THz}$ frequency. The choice of the resonant frequencies was motivated by features of the emission-the $0.3 \mathrm{THz}$ line is close to the maximal value in the red side of the spectrum, while $0.7 \mathrm{THz}$ at the blue one, as it can be seen in the measured $\mathrm{THz}$ emission spectrum results presented together with experiment schematics in Figure 5a. Sharp lines gracing with the emission spectrum are due to the water vapour absorption in the air. Spatial mode profiles recorded in parallel polarization in respect to microbolometer axis using different coupling antennas are displayed in Figure 5b,c. As it can be seen, the mode profiles can be precisely recorded using both types of antennas. The microbolometer coupled to $0.3 \mathrm{THz}$ resonant antenna removes high-frequency components of the radiation; however, its spatial profile, due to strong low-frequency constituents, reproduces the shape of the mode quite well. The distribution registered at other resonant frequency, around $0.7 \mathrm{THz}$, shows quite similar picture; however, the spatial mode distribution is narrower in comparison with that at $0.3 \mathrm{THz}$ frequency. One can presume that these data also reproduce correctly the spatial mode distribution despite of the absence of relatively strong low-frequency components recorded. The data obtained allow to infer that the antenna-coupled bolometers can be used for mode profiles control in various optoelectronic $\mathrm{THz}$ time-domain spectrometers excited by femtosecond laser pulses [27].
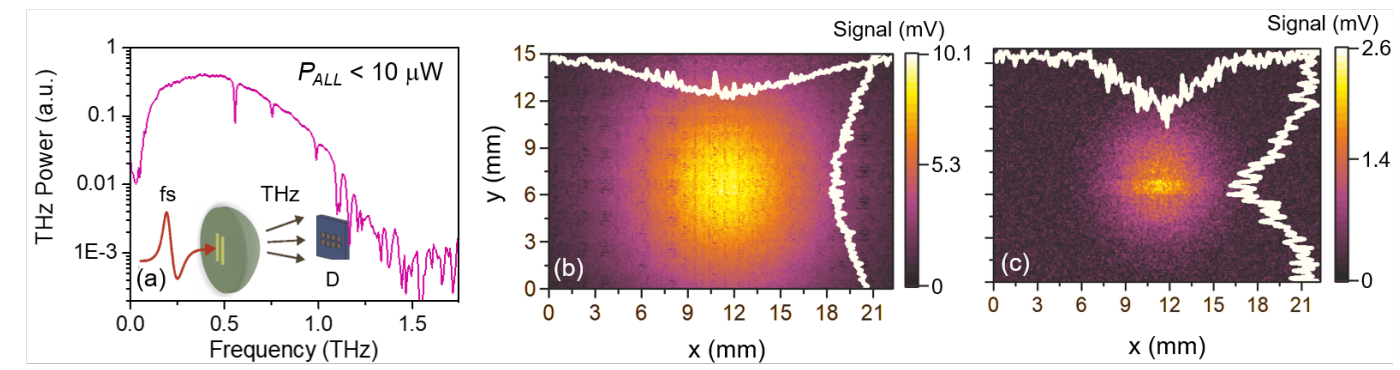

Figure 5. Spectrum of $\mathrm{THz}$ pulsed emitter (antenna switch) plotted in semi log scale (a). The measurement geometry is shown in the inset, where fs denotes to optical femtosecond pulse exciting THz emission from LTG GaAs optoelectronic switch, D labels Ti-mB detector. Note sharp lines caused by water vapour absorption in the air decorating the $\mathrm{THz}$ emission spectrum. Beam profiles of $\mathrm{THz}$ pulsed emitter obtained with antenna-coupled Ti-mB optimized for resonant frequencies of $0.3 \mathrm{THz}(\mathbf{b})$ and $0.7 \mathrm{THz}(\mathbf{c})$. White lines indicate intensity cross sections along the relevant axes.

\subsection{Mode Control in Optically-Pumped Molecular Laser}

Since the bolometers exhibit very high sensitivity, it would be of a particular interest to explore their properties far above the coupling antennas resonances. If a mode control could be possible under these unusual conditions, it could extend the device application areas into $\mathrm{THz}$ systems with a wider frequency band of operating and hence making the microbolometers more universal. In these experiments, we have chosen $\mathrm{THz}$ OPML as a source of radiation delivering discrete spectrum of $0.76 \mathrm{THz}, 1.63 \mathrm{THz}$ and $2.52 \mathrm{THz}$ at various power levels. Special attention was given to polarization-related control; it deserves to be mentioned that the spatial mode profile was intentionally disturbed by varying power of the optical pumping and adjusting grating of the pumping laser [28].

The experimental results of bolometers investigation are presented in Figure 6 together with reference data recorded by the commercially available pyroelectric detector. Emission powers at $0.76 \mathrm{THz}, 1.63 \mathrm{THz}$ and $2.52 \mathrm{THz}$ frequencies were $1.6 \mathrm{~mW}, 1.6 \mathrm{~mW}$ and $7 \mathrm{~mW}$, respectively, i.e., they were kept in a low level in comparison to conventional powers used in this type of lasers. As it can be seen, reference data presented in Figure $6 \mathrm{a}$ can be understood as the averaged signal since the detector used is insensitive to the polarization. The polarization-resolved mode structures-both for parallel and perpendicular polarizations - are displayed in panels $(b, c)$ and allow to analyse in details spatial profiles of the unfocused modes. As the diameter of the laser beam profile amounts to $11 \mathrm{~mm}$, its 
spatial deviations can be also nicely distinguished. The estimated polarization extinction ratio was found to be of $2.2 \mathrm{~dB}, 11.3 \mathrm{~dB}$ and $8.9 \mathrm{~dB}$ at $0.76 \mathrm{THz}, 1.63 \mathrm{THz}, 2.52 \mathrm{THz}$, respectively, suggesting additional application option of the Ti-mB for polarization-sensitive mode profile far above the antenna resonances. Relatively low PER value obtained at $0.76 \mathrm{THz}$ can be explained by two constituents of the mode distinctly observed in Figure 6c.

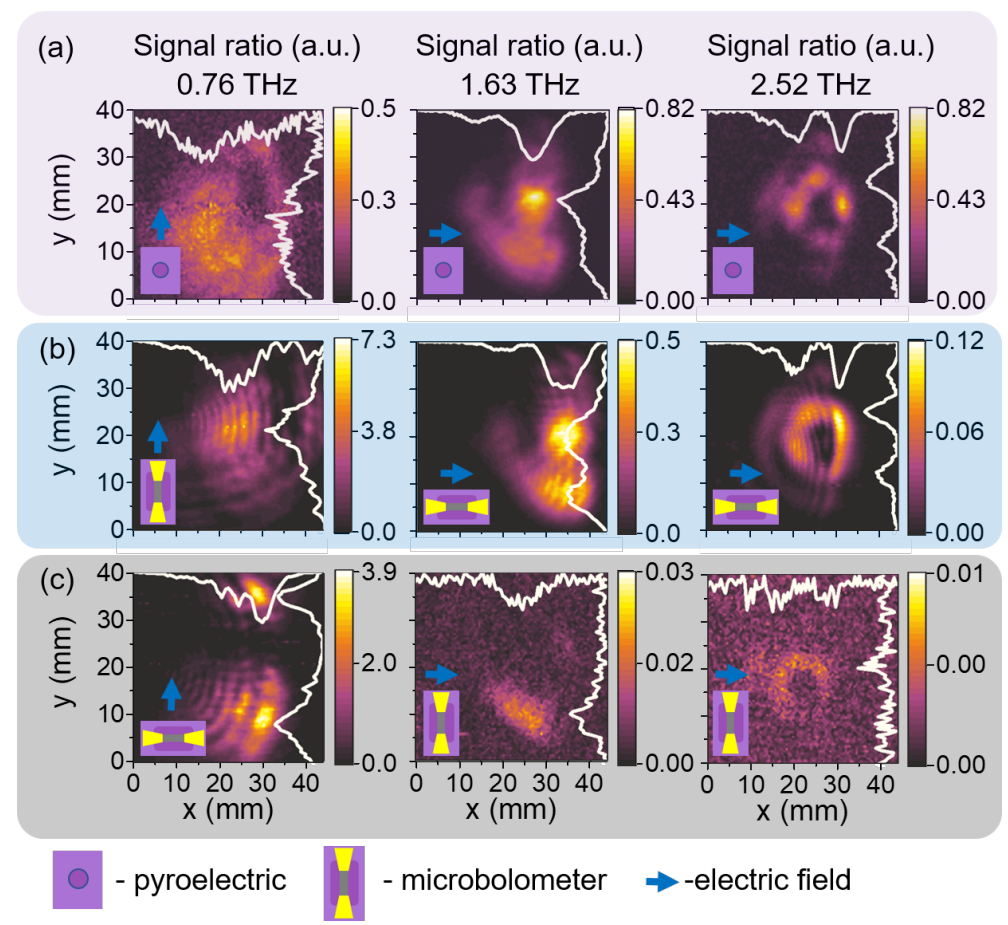

Figure 6. THz beam profiles of different polarizations obtained using optically-pumped molecular $\mathrm{THz}$ laser operating in a continuous wave mode at frequencies: $0.76 \mathrm{THz}, 1.33 \mathrm{THz}$ and $2.52 \mathrm{THz}$. Reference measurements using pyroelectric detector (a); results using microbolometer when the dipole antenna is in parallel to the electric field of the incident light (b); results using microbolometer when the dipole antenna is perpendicular to the electric field of the incident light (c). White lines indicate intensity cross sections along the relevant axes.

\subsection{Imaging with Spatial Filtering Methods}

To illustrate the sensitive operation of the developed Ti-mBs and their versatile operation using weak power sources we have proceeded in an opposite way that is usually applied in a conventional $\mathrm{THz}$ imaging. Here, as a rule, $\mathrm{THz}$ images come from scanning the object with the focused beam and registering the focused radiation on the detector making thus induced signal much larger-we have oriented our research for future applications when images without raster scanning, e.g., employing detectors arrays will be required. In such cases, the apertures of imaging optical elements become of a particular importance as the $\mathrm{THz}$ wavelength is large in comparison with diameters of optical elements and propagation distances. Therefore, a novel optical solutions [15] are needed allowing to design and manufacture compact optical elements with large aperture sizes and reasonable focal lengths. A special role can be attributed to the case of low absorbing or transparent samples, when the object observation and registration of its internal structure grow into a tremendous challenge [29]. It is related to the fact that power detectors record intensity of the radiation which suffers almost no observable change in the given circumstances, and the shift of the phase of incident radiation becomes a single quantity to be recorded.

In what follows, we employ both single pixel and arrays of the sensitive Ti-mB to record $\mathrm{THz}$ images of low absorbing objects under unfocused $\mathrm{THz}$ illumination. Both techniques-direct imaging and spatial filtering methods-are used to create a $\mathrm{THz}$ image of object inducing phase changes 
(uniform amplitude) and that can be registered by intensity sensitive Ti-mB. Conventionally, such filter must be inserted in the Fourier plane in the used imaging system and it will affect only some spatial frequencies of the Fourier spectrum of the object. One can distinguish different methods like bright and dark field [30], Schlieren [31] or phase contrast [32,33] depending on the filter type. They differ in principles and type of the filter; however, all of them permit to create an intensity pattern corresponding to phase changes in the object. The phase contrast method enables linear mapping between phase shift introduced by the object and recorded intensity pattern. In a given study we preferred a phase contrast and dark field methods to register not focused radiation with sensitive Ti-mB.

Results of the investigation together with experimental set-up are given in Figure 7. To elucidate sensing features of the microbolometers, a sample consisting of various low absorbing objects-rubber, paper towel, napkin, sponge, sponge with metal wire inside, plastic (from 1 to 4 layers), aperture of $4 \mathrm{~mm}$ in diameter, dots made of silicone, acrylic and wax as well as metal as the reference-was composed and prepared. Measurement set-up for this kind of research is depicted in Figure 7a. As one can see, it is slightly different from that displayed in Figure 2 because of the filter inserted.
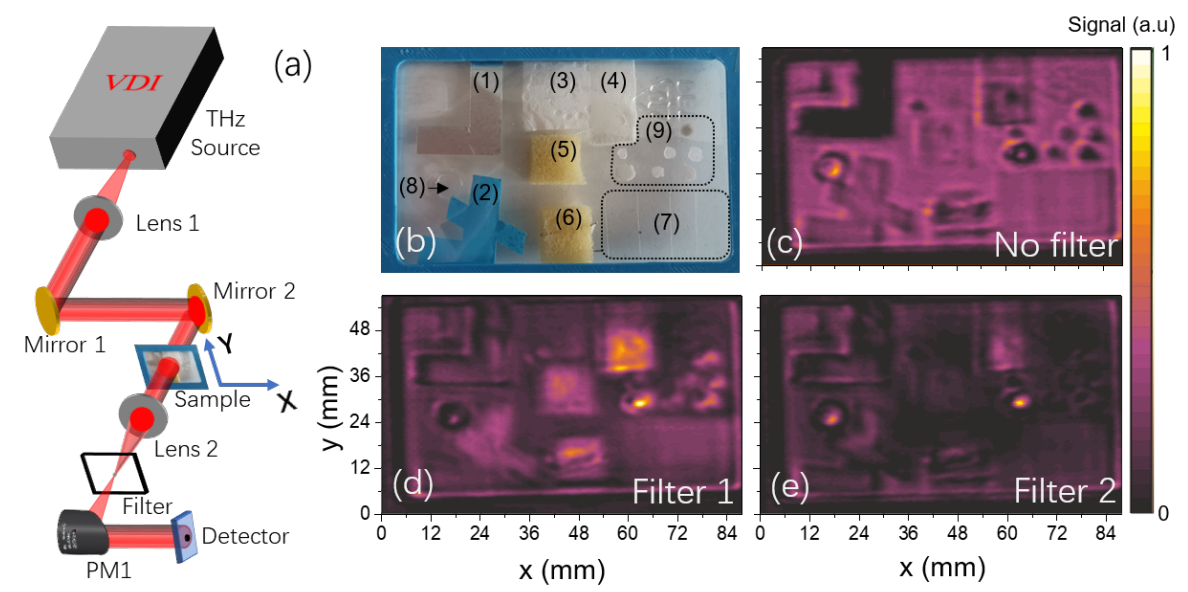

Figure 7. Principal scheme of phase contrast imaging set up for $0.3 \mathrm{THz}$, where L1-HDPE lens with $f=12 \mathrm{~cm}, \mathrm{M} 1, \mathrm{M} 2$ - gold plated flat mirrors, sample, L2-TPX lens with $f=6 \mathrm{~mm}$, Filter-(1): $\oslash=2 \mathrm{~mm}$ circle shaped four paper sheets, (2)- $\oslash=2 \mathrm{~mm}$ circle shaped aluminum foil, PM1—off axis parabolic mirror with $f=5 \mathrm{~cm}$ (a). Photo of the sample with plastic substrate film, it consists of: (1)—metal, (2)—rubber, (3)—paper towel, (4)—napkin, (5)— sponge, (6)—sponge with metal wire inside, (7)—plastic (from 1 to 4 layers), (8)—aperture (diameter: $4 \mathrm{~mm}$ ), (9)—dots, made of silicone, acrylic and wax (b). Direct raster scan image of the sample at $0.3 \mathrm{THz}(\mathbf{c})$. Phase contrast images obtained using filter $1(\mathbf{d})$ and filter $2(\mathbf{e})$. THz image pixel size: $0.3 \mathrm{~mm} \times 0.3 \mathrm{~mm}$; images consist of $292 \times 190$ pixels.

Two types of filters were used in the experiments: the filter 1, consisting of $2 \mathrm{~mm}$ circle shaped four paper sheets, was a phase filter introducing a phase retardation of $\pi / 2$ only in the central part of the Fourier spectrum and enabling thus to create an image using phase contrast method; second one, filter 2, circle shaped aluminum foil of $2 \mathrm{~mm}$ diameter, was an amplitude filter, blocking low spatial frequencies, which resulted in creating an intensity image of phase object having dark background (which is related to removal of low spatial frequencies).

Sample photo and its direct image as well as two corresponding intensity images of phase objects are shown in the corresponding panels $(b-d)$ of Figure 7 . It can be easily seen that even direct imaging nicely resolves all the objects illustrating suitability of Ti-mB for imaging of low absorbing objects. As for deeper insight, transparent objects like sponge ( 5 and 6 ) and paper tissue (4) are much better visible in case of phase contrast method (filter 1), whilst dark field method (filter 2) gives much better results for imaging of thin rubber glove (2), where we can clearly see edges of the rubber shape. 
Finally, it is worth to notice the operation speed of the device. Earlier it was determined that the Ti-mB coupled with a $0.3 \mathrm{THz}$ frequency dipole antenna exhibited the response time of $1 \mu$ s range and maintained a sensitivity of $300 \mathrm{~V} / \mathrm{W}$ and NEP as low as $14 \mathrm{pW} / \sqrt{\mathrm{Hz}}$ [26]. If compared with other similar devices based on thermoelectricity-related phenomena, for instance, with uncooled antenna-coupled terahertz detectors based on $\mathrm{BiSb} / \mathrm{Sb}$ thermocouples with $22 \mu \mathrm{s}$ response time and NEP of $170 \mathrm{pW} / \sqrt{\mathrm{Hz}}$ [34] and bow-tie diodes operating on hot-electrons effects with sensitivity of $5 \mathrm{~V} / \mathrm{W}, \mathrm{NEP}$ of $5.8 \mathrm{nW} / \sqrt{\mathrm{Hz}}$ and response time less than $7 \mathrm{~ns}$ [35], the described titanium-based microbolometers with readout electronics exhibit high sensitivity exceeding $200 \mathrm{kV} / \mathrm{W}$ and relatively short response time ( 5 microseconds including electronics circuit). Therefore, these parameters together with electronics that can work up to $200 \mathrm{ksps}$ (kilo samples per second) simultaneous sampling speed, makes the Ti-mB promising for real-time fine optical adjustment and mode profile control aims in $\mathrm{THz}$ spectroscopic and imaging systems as well as imaging applications and inspection of low-absorbing objects.

\section{Materials and Methods}

Each microbolometer was fabricated from titanium, which was chosen among several appropriate materials, as its micromachining properties were found to be most suitable for silicon processing technology, temperature coefficient and reliability. The sensor was processed on the silicon nitride (SiN) membrane of $2 \mu \mathrm{m}$ thickness; subsequently, the titanium thermistor film is electro-deposited onto the membrane, along with the aluminum antenna and metal interconnections. The thermistor is suspended in the air by etching out the underlying membrane. The microbolometer was connected either to double-dipole or to log-periodic type $\mathrm{THz}$ antenna [23]. Both single pixel bolometers and their linear arrays consisting of 32 pixels were used in the experiments, where pixel size was $2.5 \times 2.5 \mathrm{~mm}^{2}$, pixel pitch was $2.5 \mathrm{~mm}$. Just one pixel of the linear array was active during all experiments. Sensitivity and NEP of the arrays of sensors exceeds $200 \mathrm{kV} / \mathrm{W}$ and less then $20 \mathrm{pW} / \sqrt{\mathrm{Hz}}$, respectively. Linear arrays of titanium microbolometers were produced by Luvitera, Ltd, Vilnius, Lithuania.

Electronic multiplier sources (Virginia Diodes, Inc., Charlottesville, Virginia, United States of America) were used to generate radiation of $0.15 \mathrm{THz}, 0.3 \mathrm{THz}$ and $0.6 \mathrm{THz}$ frequencies in continuous wave mode with power of $29 \mathrm{~mW}, 13.2 \mathrm{~mW}$ and $0.56 \mathrm{~mW}$, respectively.

Commercially available frequency-domain terahertz spectrometer (Toptica Terascan 780) based on GaAs photo-mixer coupled with silicon lens was engaged in the experiments. Its operation range is within $0.1-2 \mathrm{THz}$, it delivers radiation of circular polarization of about $1 \mu \mathrm{W}$ power.

For the experiment a broadband photoconductive low-temperature grown gallium arsenide (LTG-GaAs) antenna coupled with silicon lens from time-domain spectrometer (Teravil, Ltd., Vilnius, Lithuania) was also used. The microbolometer was connected to the lock-in amplifier to modulate signal optically chopped at frequency of $500 \mathrm{kHz}$. The emitter was pumped with femtosecond Ti-Sapphire optical laser of $100 \mathrm{fs}$ pulse duration and generating broadband $\mathrm{THz}$ pulses in frequency range of $0.1-2.5 \mathrm{THz}$ with average emission power of $1.2 \mu \mathrm{W}$. The $\mathrm{THz}$ beam was investigated by scanning it in the $x y$ plane perpendicular to the direction of propagation with detector being fixed to $x y$ motorized positioning stage. No additional focusing optical components are used in radiation measurements. In beam imaging recording, position synchronized raster scan technique was implemented similar to that described in Ref. [9]. Scanning parameters were selected to enable sufficient SNR with resolution up to $0.1 \times 0.25 \mathrm{~mm}^{2}$ which is comparable with the wavelength of upper frequency limit of emitted radiation (or the size of the microbolometer).

Optically pumped continuous-wave molecular THz laser FIRL-100 (Edinburg Instruments, Ltd., Livingston, United Kingdom) was used to generate discrete spectrum at $0.76 \mathrm{THz}, 1.63 \mathrm{THz}$ and $2.52 \mathrm{THz}$ frequencies with corresponding powers of $1.6 \mathrm{~mW}, 1.6 \mathrm{~mW}$ and $7 \mathrm{~mW}$, respectively. The emitted unfocused radiation was of $11 \mathrm{~mm}$ in diameter; the power was artificially reduced varying the power of optical pumping from $20 \mathrm{~W}$ down to $9.8 \mathrm{~W}$ range. 
The absolute power was measured using a calibrated power meter (Thomas Keating Absolute Power Meter System, Version 2, Thomas Keating Ltd., Billingshurst, West Sussex, UK).

Commercially available pyroelectric sensor SPH62 THz with $2 \mathrm{~mm}^{2}$ active area from Spectrum Detector Inc. was used in the experiments.

All experiments were conducted in the ambient air conditions.

\section{Conclusions}

Versatile operation of universal, convenient and easy-to-use antenna coupled sensitive titanium-based microbolometers dedicated to the precise alignment and the control of spatial mode profiles without additional focusing optical components of weak power $\mathrm{THz}$ sources are demonstrated. Spatial mode profile control and polarization-resolved mode structures are recorded for different type $\mathrm{THz}$ emitters-electronic multiplier sources, optical $\mathrm{THz}$ mixers-based frequency domain and femtosecond optoelectronic $\mathrm{THz}$ time-domain spectrometers as well as optically pumped molecular $\mathrm{THz}$ laser-operating either in continuous wave or pulsed modes. Features of the microbolometers coupled with resonant antennas within $0.15-0.6 \mathrm{THz}$ range are exposed and discussed, and their ability to detect spatial mode profiles far above the antennas resonances, up to $2.54 \mathrm{THz}$, are explored as well. It is found that a broadband antenna (below $1 \mathrm{THz}$ ) coupled microbolometer reproduces well the spatial mode profile of time-domain spectrometer. Polarization-sensitive mode control possibilities are examined in details; suitability of the resonant antenna-coupled bolometers to resolve low-absorbing objects at $0.3 \mathrm{THz}$ is revealed via direct, dark field and phase contrast imaging techniques. The microbolometer, for instance, coupled with a $0.3 \mathrm{THz}$ frequency dipole antenna exhibits the response time of $1 \mu$ s range, sensitivity of $300 \mathrm{~V} / \mathrm{W}$, and noise-equivalent power of $14 \mathrm{pW} / \sqrt{\mathrm{Hz}}$. Microbolometers display corresponding values of $200 \mathrm{kV} / \mathrm{W}$ and less then $20 \mathrm{pW} / \sqrt{\mathrm{Hz}}$, respectively, and the response time in the $5 \mu$ s range. These parameters make the devices promising for real-time fine adjustment of $\mathrm{THz}$ spectroscopic and imaging systems as well as imaging applications and inspection of low-absorbing objects.

Author Contributions: Conceptualization of the research, G.V. and L.M.; methodology, G.V., L.M. and A.S. (Agnieszka Siemion); software, D.J.; development of antenna-coupled Ti-microbolometers, A.S. (Aleksander Sešek), A.Š., I.K., and J.T.; investigation using electronic sources, L.M., A.S. (Agnieszka Siemion), L.Q. and D.J.; investigation using frequency domain spectrometer, D.S.; investigation using THz time-domain spectrometer, I.K.; investigation using optically-pumped molecular THz laser, D.S., L.Q., and L.M.; data acquisition, L.M. and G.V.; experimental data analysis, L.M., A.S. (Agnieszka Siemion), L.Q., and G.V.; writing-original draft preparation, G.V.; writing-review and editing, L.M., A.S. (Agnieszka Siemion), I.K, and G.V.; visualization, L.M., A.S. (Agnieszka Siemion), L.Q., and D.J.; supervision and coordination, G.V.; project administration, G.V. All authors have read and agreed to the published version of the manuscript.

Funding: L.M. and I.K. acknowledge financial support from "KOTERA-PLAZA" project supported from European Regional Development Fund (Grant No. 01.2.2-LMT-K-718-01-0047).

Acknowledgments: Authors are very grateful to Rimvydas Venckevičius and Ramūnas Adomavičius for their kind assistance in experiments with femtosecond optoelectronic THz emitters and for illuminating discussions.

Conflicts of Interest: The authors declare no conflict of interest.

\section{Abbreviations}

The following abbreviations are used in this manuscript:

$\begin{array}{ll}\text { THz } & \text { Terahertz } \\ \text { THz ELS } & \text { Terahertz electronic multiplier source } \\ \text { THz FDS } & \text { Terahertz frequency domain spectrometer } \\ \text { THz TDS } & \text { Terahertz time domain spectrometer } \\ \text { THz OPML } & \text { Optically pumped molecular THz laser } \\ \text { Ti-mB } & \text { titanium-based microbolometer } \\ \text { CW } & \text { continuous wave } \\ \text { SNR } & \text { Signal-to-noise ratio } \\ \text { Ksps } & \text { Kilo samples per second }\end{array}$




\section{References}

1. Mittleman, D.M. Twenty years of terahertz imaging [Invited]. Opt. Express 2018, 26, 9417. [CrossRef] [PubMed]

2. Son, J.H.; Oh, S.J.; Cheon, H. Potential clinical applications of terahertz radiation. J. Appl. Phys. $2019,125$. [CrossRef]

3. Puc, U.; Abina, A.; Jeglič, A.; Zidanšek, A.; Kašalynas, I.; Venckevičius, R.; Valušis, G. Spectroscopic Analysis of Melatonin in the Terahertz Frequency Range. Sensors 2018, 18, 4098. [CrossRef]

4. Sun, Q.; He, Y.; Liu, K.; Fan, S.; Parrott, E.P.; Pickwell-MacPherson, E. Recent advances in terahertz technology for biomedical applications. Quant. Imaging Med. Surg. 2017, 7, 345-355. [CrossRef] [PubMed]

5. Trofimov, V.A.; Varentsova, S.A. A possible way for the detection and identification of dangerous substances in ternary mixtures using thz pulsed spectroscopy. Sensors 2019, 19, 2365. [CrossRef] [PubMed]

6. Palka, N.; Szala, M. Transmission and Reflection Terahertz Spectroscopy of Insensitive Melt-Cast High-Explosive Materials. J. Infrared Millimeter Terahertz Waves 2016, 37, 977-992. [CrossRef]

7. Puc, U.; Abina, A.; Rutar, M.; Zidanšek, A.; Jeglič, A.; Valušis, G. Terahertz spectroscopic identification of explosive and drug simulants concealed by various hiding techniques. Appl. Opt. 2015, 54, 4495. [CrossRef] [PubMed]

8. Bauer, M.; Venckevičius, R.; Kašalynas, I.; Boppel, S.; Mundt, M.; Minkevičius, L.; Lisauskas, A.; Valušis, G.; Krozer, V.; Roskos, H.G. Antenna-coupled field-effect transistors for multi-spectral terahertz imaging up to 4.25 THz. Opt. Express 2014, 22, 19235. [CrossRef]

9. Kasalynas, I.; Venckevicius, R.; Valusis, G. Continuous Wave Spectroscopic Terahertz Imaging With InGaAs Bow-Tie Diodes at Room Temperature. IEEE Sens. J. 2013, 13, 50-54. [CrossRef]

10. Minkevičius, L.; Tamošiūnas, V.; Kašalynas, I.; Seliuta, D.; Valušis, G.; Lisauskas, A.; Boppel, S.; Roskos, H.G.; Köhler, K. Terahertz heterodyne imaging with InGaAs-based bow-tie diodes. Appl. Phys. Lett. 2011, 99, 131101. [CrossRef]

11. Murate, K.; Kawase, K. Perspective: Terahertz wave parametric generator and its applications. J. Appl. Phys. 2018, 124, 160901. [CrossRef]

12. Trofimov, V.A.; Varentsova, S.A.; Zakharova, I.G.; Zagursky, D.Y. New possibilities of substance identification based on $\mathrm{THz}$ time domain spectroscopy using a cascade mechanism of high energy level excitation. Sensors 2017, 17, 2728. [CrossRef]

13. Abina, A.; Puc, U.; Jeglič, A.; Prah, J.; Venckevičius, R.; Kašalynas, I.; Valušis, G.; Zidanšek, A. Qualitative and quantitative analysis of calcium-based microfillers using terahertz spectroscopy and imaging. Talanta 2015, 143, 169-177. [CrossRef] [PubMed]

14. Tamošiunas, V.; Minkevičius, L.; Vaitkunas, A.; Urbanowicz, A.; Maneikis, A.; Šetkus, A.; Valušis, G. Characterization of Silicon Solar Cells and Module Materials Using Terahertz Radiation. Energy Procedia 2015, 77, 202-207. [CrossRef]

15. Siemion, A. Terahertz Diffractive Optics-Smart Control over Radiation. J. Infrared Millimeter Terahertz Waves 2019, 40, 477-499. [CrossRef]

16. Molloy, J.F.; Naftaly, M.; Dudley, R.A. Characterization of terahertz beam profile and propagation. IEEE J. Sel. Top. Quantum Electron. 2013, 19, 8401508. [CrossRef]

17. Bitman, A.; Lumer, Y.; Moshe, I.; Zalevsky, Z. Characterization of spectrally broadband terahertz beam propagation. J. Opt. Soc. Am. B 2012, 29, 1436. [CrossRef]

18. Molloy, J.; Naftaly, M.; Dudley, R. Characterisation of Terahertz Beam Profile and Propagation through Complex Quasi-Optic Systems. Terahertz Sci. Technol. 2011, 4, 99-103. [CrossRef]

19. Jokubauskis, D.; Minkevičius, L.; Karaliūnas, M.; Indrišiūnas, S.; Kašalynas, I.; Račiukaitis, G.; Valušis, G. Fibonacci terahertz imaging by silicon diffractive optics. Opt. Lett. 2018, 43, 2795. [CrossRef]

20. Minkevičius, L.; Jokubauskis, D.; Kašalynas, I.; Orlov, S.; Urbas, A.; Valušis, G. Bessel terahertz imaging with enhanced contrast realized by silicon multi-phase diffractive optics. Opt. Express 2019, 27, 36358. [CrossRef]

21. Oda, N. Détecteur matriciel de type bolométrique à température ambiante et camera vidéo pour l'imagerie térahertz. C. R. Phys. 2010, 11, 496-509. [CrossRef]

22. Oden, J.; Meilhan, J.; Lalanne-Dera, J.; Roux, J.F.; Garet, F.; Coutaz, J.L.; Simoens, F. Imaging of broadband terahertz beams using an array of antenna-coupled microbolometers operating at room temperature. Opt. Express 2013, 21, 4817. [CrossRef] [PubMed] 
23. Trontelj, J.; Valušis, G.; Venckevičius, R.; Kašalynas, I.; Sešek, A.; Švigelj, A. A high performance room temperature THz sensor. Terahertz Emit. Receiv. Appl. V 2014, 9199, 91990K. [CrossRef]

24. Sešek, A.; Švigelj, A.; Trontelj, J.; A compact THz imaging system. In Image Sensors and Imaging Systems 2015; Widenhorn, R., Dupret, A., Eds.; SPIE: San Francisco, CA, USA, 2015; pp. 7-14. [CrossRef]

25. Trontelj, J.; Sešek, A.; Electronic terahertz imaging for security applications. In Terahertz, RF, Millimeter, and Submillimeter-Wave Technology and Applications IX; Sadwick, L.P., Yang, T., Eds.; SPIE: San Francisco, CA, USA, 2016; pp. 161-166. [CrossRef]

26. Kašalynas, I.; Venckevičius, R.; Minkevičius, L.; Sešek, A.; Wahaia, F.; Tamošiūnas, V.; Voisiat, B.; Seliuta, D.; Valušis, G.; Švigelj, A.; et al. Spectroscopic Terahertz Imaging at Room Temperature Employing Microbolometer Terahertz Sensors and Its Application to the Study of Carcinoma Tissues. Sensors 2016, 16, 432. [CrossRef]

27. Krotkus, A. Semiconductors for terahertz photonics applications. J. Phys. D Appl. Phys. 2010, 43, 273001. [CrossRef]

28. Kašalynas, I.; Venckevičius, R.; Tumonis, L.; Voisiat, B.; Seliuta, D.; Valušis, G.; Račiukaitis, G. Reflective terahertz imaging with the TEM01 mode laser beam. Appl. Opt. 2013, 52, 5640-5644. [CrossRef]

29. Jokubauskis, D.; Minkevičius, L.; Seliuta, D.; Kašalynas, I. Terahertz homodyne spectroscopic imaging of concealed low-absorbing objects. Opt. Eng. 2019, 58, 023104. [CrossRef]

30. Hall, C.E. Dark-field electron microscopy. I. Studies of crystalline substances in dark-field. J. Appl. Phys. 1948, 19, 198-212. [CrossRef]

31. Hargather, M.J.; Settles, G.S. Natural-background-oriented schlieren imaging. Exp. Fluids 2010, 48, 59-68. [CrossRef]

32. Zernike, F. Diffraction theory of the knife-edge test and its improved form, the phase-contrast method. Mon. Not. R. Astron. Soc. 1934, 94, 377-384. [CrossRef]

33. Zernike, F. Diffraction theory of the knife-edge test and its improved form: The phase-contrast method. J. Micro/Nanolithogr. MEMS MOEMS 2002, 1, 87-95. [CrossRef]

34. Huhn, A.K.; Spickermann, G.; Ihring, A.; Schinkel, U.; Meyer, H.G.; Haring Bolívar, P. Uncooled antenna-coupled terahertz detectors with $22 \mu$ s response time based on BiSb/Sb thermocouples. Appl. Phys. Lett. 2013, 102, 121102. [CrossRef]

35. Kašalynas, I.; Seliuta, D.; Simniškis, R.; Tamošiunas, V.; Köhler, K.; Valušis, G. Terahertz imaging with bow-tie InGaAs-based diode with broken symmetry. Electron. Lett. 2009, 45, 833-835. [CrossRef] 